\title{
Influence of Finite Element Size in Residual Strength Prediction of Composite Structures
}

\author{
Arunkumar Satyanarayana ${ }^{1}$ \\ ATK Space Division, Hampton, VA, 23681-2199 \\ Philip Bogert ${ }^{2}$ \\ NASA Langley Research Center, Hampton, VA 23681-2199 \\ Kazbek.Z.Karayev, ${ }^{3}$ Paul.S.Nordman ${ }^{4}$ and Hamid Razi ${ }^{3}$ \\ The Boeing Company, Seattle WA 98124-2207
}

\begin{abstract}
The sensitivity of failure load to the element size used in a progressive failure analysis (PFA) of carbon composite center notched laminates is evaluated. The sensitivity study employs a PFA methodology previously developed by the authors consisting of HashinRotem intra-laminar fiber and matrix failure criteria and a complete stress degradation scheme for damage simulation. The approach is implemented with a user defined subroutine in the ABAQUS/Explicit finite element package. The effect of element size near the notch tips on residual strength predictions was assessed for a brittle failure mode with a parametric study that included three laminates of varying material system, thickness and stacking sequence. The study resulted in the selection of an element size of $0.09 \mathrm{in}$. X 0.09 in., which was later used for predicting crack paths and failure loads in sandwich panels and monolithic laminated panels. Comparison of predicted crack paths and failure loads for these panels agreed well with experimental observations. Additionally, the element size vs. normalized failure load relationship, determined in the parametric study, was used to evaluate "strength-scaling" factors for three different element sizes. The failure loads predicted with all three element sizes provided converged failure loads with respect to that corresponding with the $0.09 \mathrm{in}$. $X 0.09$ in. element size. Though preliminary in nature, the strength-scaling concept has the potential to greatly reduce the computational time required for PFA and can enable the analysis of large scale structural components where failure is domiminated by fiber failure in tension.
\end{abstract}

\section{Introduction}

I $\mathrm{n}$ recent years, progressive failure analysis (PFA) methodologies have been used widely in research organizations and are starting to be used by industry for assessment of the residual strength of composite structures. Using the PFA methodologies, many researchers have been able to simulate various damage modes such as fiber and matrix failures within plies and also delamination between the plies. PFA methodologies, in general, incorporate stress or strain based failure criteria to identify the onset of damage in conjunction with a material degradation process to simulate damage progression. A virtual through the thickness crack in a finite element model, representing the actual structural component, can be created by using the damage progression algorithm along with the element deletion option available in most explicit finite element solvers.

Even though PFA methodologies have been used extensively in simulating damage progression and ultimately the failure of structures, the predicted failure loads are highly sensitive to element size. This issue has been addressed in Refs. 1- 4 by equating the critical fracture energies of the material to the energy dissipation due to the damage mode. This procedure has the inherent tendency to allow for adjusting the elements' strengths based on their size.

\footnotetext{
${ }^{1}$ Senior Structural Analyst, NASA Langley Research Center, Mail Stop 190.

${ }^{2}$ Research Aerospace Engineer, Structural Mechanics and Concepts Branch, 8 West Taylor St., Mail Stop 190.

${ }^{3}$ Associated Technical Fellow, The Boeing Company, Seattle, WA.

${ }^{4}$ Senior Engineer, The Boeing Company, Seattle, WA.
} 
A simpler alternative approach to overcoming element size sensitivity in predicting failure loads has been used in Refs. 5-7. In these studies, a parametric study was conducted to select an element size around the singular regions in the structure which yielded a failure load within a few percent of experimental results for a particular type of coupon. The same element size was then used in the development of finite element models of other coupons or panels with varying geometric dimensions, layup and material systems. The element size determined by Coats and Harris (Ref. 5) is different than that used by the present authors in Ref. 6. As the authors analyzed larger coupons and different configurations it was found that the element size previously determined by the authors in Ref. 6 worked well for most configurations but for some, failure loads were not predicted accurately. It was concluded that perhaps an appropriate element size had not been found. Encouraged by the earlier success, a numerical experiment was conducted to study element size effects in more detail and to identify the range of finite element sizes that would predict the residual strength of the composite structures. The element size, obtained from that numerical experiment, was found to be slightly different than the one used previously in Ref. 6 . The newly determined element size was then adopted to develop finite element models of a number of other configurations containing both notches and holes, ${ }^{7}$ including those in which some loads had been predicted previously. Use of the new element size produced results which were found to be in better agreement with test data. Though the element orientation can also play an important role in the determination of failure loads and modes it has not been addressed here. These effects have been considered in detail in Ref. 8. Additionally, with the current approach it was observed that for the range of element sizes under consideration, the use of elements aligned orthogonally with the notch was capable of predicting angular damage growth, when through cracks or through splits were observed, especially in larger panels.

In the present paper, a summary of the PFA methodology previously developed by the authors is presented as background. Next, a description of a numerical experiment conducted to determine the element size required to perform PFA of notched coupons in which failure is dominated by a single failure mode, is provided. Having determined the element size for use in PFA, it is then applied to a variety of notched compression and tension panels fabricated and tested by the Boeing Company during the last several years. The description of the panels and coupons analyzed is provided along with the failure loads and crack trajectories determined analytically and experimentally. Finally, an approach, using 'strength-scaling' factors, is investigated in order to potentially overcome the sensitivity of element size on the failure load predictions where the damage is dominated by fiber failures. The goal of this investigation is to potentially increase the minimum required element size for PFA for improved computational efficiency, which quickly becomes a limiting factor on large and built-up structures. Preliminary findings in this area are reported.

\section{Progressive Failure Analysis Methodology - In-Plane Damage Initiation, Degradation Model and Damage Evolution}

Progressive failure analysis of center-notched coupons and large center-notched sandwich and laminated composite panels is performed using the ABAQUS ${ }^{\mathrm{TM}} /$ Explicit $^{9}$ finite element code with the damage model described below to simulate the intra-laminar damage modes. This damage model, which simulates in-plane damage modes such as fiber and matrix failures, is applicable to two dimensional shell elements only. Unidirectional failure criteria have been used to identify the onset of damage at a material point and a plydegradation approach ${ }^{10}$ has been used for the damage evolution process. The entire procedure has been implemented using the user written subroutine $\operatorname{VUMAT}^{9}$ to avoid the numerical convergence issues encountered with implicit codes when deleting elements to simulate through-thickness cracks. The damage evolution procedure adopted in our methodology is slightly different than the standard built-in damage model in the ABAQUS ${ }^{9}$ code. This damage model is applicable in performing PFA of composite structures subjected to uni-axial loading conditions such as tension or compression and has not been verified for bi-axial loading conditions.

In the current damage model, the Hashin-Rotem unidirectional failure criteria ${ }^{11}$ are used to detect fiber and matrix damage in a lamina. The failure criteria are expressed in terms of the in-plane stresses $\sigma_{i j}$, the strengths $X$ and $Y$, and the shear strength $S$. The in-plane stresses $\sigma_{11}, \sigma_{22}$, and $\sigma_{12}$, are aligned with the material coordinate system in the ply, with $\sigma_{11}$ parallel to the fibers. The strengths parallel and transverse to the fiber direction are denoted as $X$ and $Y$, respectively. The in-plane shear strength is denoted as $S_{12}$. The subscripts $c$ and $t$ denote compression and tension, respectively. The Hashin-Rotem failure criteria are described below.

The fiber failure index in tension for a $\sigma_{11}$ stress greater than or equal to zero is defined as:

$$
F_{f}^{T}=\left(\frac{\sigma_{11}}{X_{t}}\right)
$$


The fiber failure index in compression for a $\sigma_{11}$ stress less than zero is defined as:

$$
F_{f}^{C}=\left(\frac{\sigma_{11}}{X_{c}}\right)
$$

Similarly the matrix failure index in tension for a $\sigma_{22}$ greater than or equal to zero is defined as:

$$
M_{f}^{T}=\left(\frac{\sigma_{22}}{Y_{t}}\right)^{2}+\left(\frac{\sigma_{12}}{S_{12}}\right)^{2}
$$

The matrix failure index in compression for a $\sigma_{22}$ less than zero is defined as:

$$
M_{f}^{C}=\left(\frac{\sigma_{22}}{Y_{c}}\right)^{2}+\left(\frac{\sigma_{12}}{S_{12}}\right)^{2}
$$

In the current methodology, two damage variables $d_{F}$ and $d_{M}$ are used to represent the fiber and matrix damage modes, respectively. Each damage variable has the value of zero (no damage) or 1.0 (complete damage). When either $F_{f}^{T}$ or $F_{f}^{C}$ reaches a value greater than or equal to 1.0 , the fiber damage variable $d_{F}$ is assigned a value of 1.0. Similarly when either $M_{f}^{T}$ or $M_{f}^{C}$ reaches a value greater than or equal to 1.0, the matrix damage variable $d_{M}$ is assigned a value of 1.0. The damage variables $d_{F}$ and $d_{M}$ remain zero when the fiber and matrix failure indices $\left(F_{f}^{T}, F_{f}^{C}, M_{f}^{T}\right.$ and $M_{f}^{C}$ ) are less than 1.0.

The constitutive stress-strain relationship of a damaged ply is defined as

$$
\left\{\begin{array}{l}
\sigma_{11} \\
\sigma_{22} \\
\sigma_{12}
\end{array}\right\}=\left[\begin{array}{ccc}
\left(1-d_{F}\right) Q_{11} & \left(1-d_{F}\right)\left(1-d_{M}\right) Q_{12} & 0 \\
\left(1-d_{F}\right)\left(1-d_{M}\right) Q_{12} & \left(1-d_{F}\right)\left(1-d_{M}\right) Q_{22} & 0 \\
0 & 0 & \left(1-d_{F}\right)\left(1-d_{M}\right) Q_{66}
\end{array}\right]\left\{\begin{array}{l}
\varepsilon_{11} \\
\varepsilon_{22} \\
\varepsilon_{12}
\end{array}\right\}
$$

where

$$
\begin{aligned}
& Q_{11}=\frac{E_{1}}{\left(1-\left(1-d_{M}\right) v_{12} v_{21}\right)} \\
& Q_{12}=\frac{v_{12} E_{2}}{\left(1-\left(1-d_{M}\right) v_{12} v_{21}\right)} \\
& Q_{22}=\frac{E_{2}}{\left(1-\left(1-d_{M}\right) v_{12} v_{21}\right)} \\
& Q_{66}=G_{12}
\end{aligned}
$$

The model adopted to degrade the axial, transverse and shear stresses, once they reach their failure limits at a material point, is shown in Fig. 1. From the constitutive Eq. (5), it can be noticed that when the damage parameter $d_{M}$ attains a value of 1.0 both the transverse and the shear stresses are reduced to zero instanteneously. This type of instantaneous stress degradation to zero is characterized as a single-step or instantaneous degradation approach. Similarly, when the damage parameter $d_{F}$ attains a value of 1.0, all of the in-plane stresses at a material point become zero. When a fiber failure is detected, the axial stress at the material point is degraded either instanteneously, linearly or following a polynominal path for a small duration of time to ensure numerical stability (see Fig. 1a). When a higher order polynomial curve is adopted to degrade the axial stress, numerical oscillations in 
the solutions can be reduced. However, the transverse and shear stresses are decreased to a zero stress level instantaneously. Another characteristic of this damage model which is different from the ABAQUS ${ }^{9}$ damage model is the complete elimination of the influence of Poisson's ratio $(v)$ in evaluating the axial stress in the fibers when the matrix has failed due to transverse or shear stress (see Eq. (6)). This stress degradation model is referred to as the COmplete STress Reduction (COSTR) damage model in the remainder of the paper.

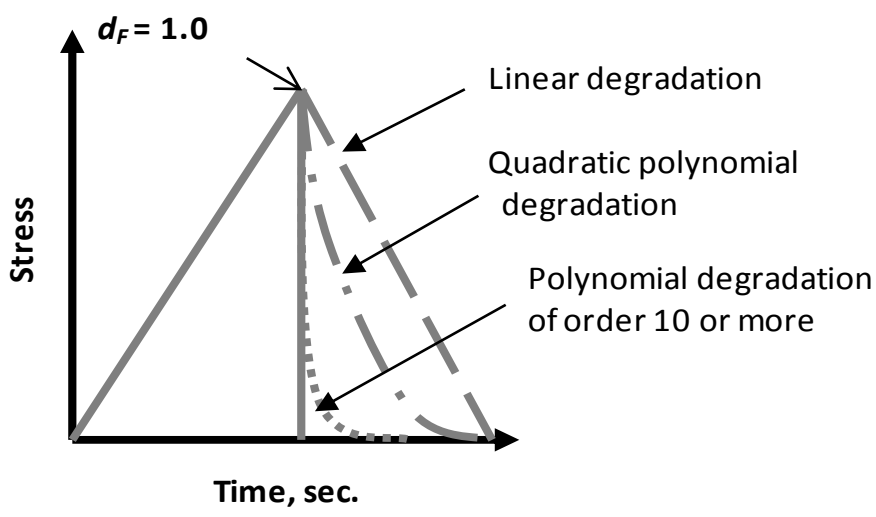

a) Axial Stress.

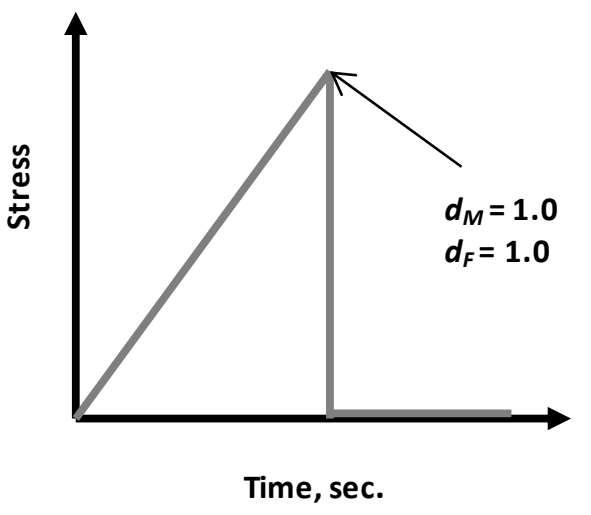

b) Transverse and Shear Stresses.

Figure 1. Stresses degradation approach used in COSTR damage model.

It has been observed that in some plies of a laminate, depending upon the loading conditions, the transverse strain, shear strain or the combination of transverse and shear strains could result in significant deformation causing excessive distortion of the element without significant stress in the fiber direction. An example of this phenomenon could be noticed in a laminate consisting of $90^{\circ}$ plies and loaded in the direction perpendicular to the fibers. To avoid excessive distortion of an element due to large strains in the matrix, the quadratic strain criterion as represented in Eq. (7) was formulated and implemented in the damage evolution procedure of the PFA methodology. Once the matrix strain failure criterion (MSFC) at a material point reaches $25 \%$, the material point is deleted. Material points are also deleted when fiber failure is detected. Once a material point is deleted it cannot be reactivated. The ABAQUSTM/Explicit ${ }^{9}$ solver checks for elements where all of the material points have been flagged as deleted and removes these elements from further computations, thereby simulating virtual cracks in the finite element model. The failure detection criteria, the COSTR damage model and the element removal procedure are all implemented using a user-written subroutine, VUMAT ${ }^{9}$ as mentioned earlier in this section. In the current study, PFA of notched coupons and large notched panels, which will be presented in later sections, are performed by instantaneously degrading the fiber direction stress to zero when the damage variable $\boldsymbol{d}_{\boldsymbol{F}}$ becomes 1.0.

$$
M S F C=\sqrt{\left(\varepsilon_{22}\right)^{2}+\left(\gamma_{12}\right)^{2}}
$$

\section{Effect of Element Size on Strength Prediction}

The sensitivity of failure load to element size around stress concentrations in a finite element model (FEM) makes predicting residual strength of composite structures very challenging. A numerical experiment was conducted on a center-notched coupon which is shown in Fig. 2, to study the influence of element size on the failure load predictions where the damage is dominated by fiber failures and other damage interactions are not considered. The center-notched coupon used in this study is $3.0 \mathrm{in}$. wide and $6.0 \mathrm{in}$. long with a notch length of $0.75 \mathrm{in}$. One of the shorter edges of the coupon is fixed in all degrees of freedom (dof). The other shorter edge is fixed in all dof except the axial direction. A uniform end displacement in the axial direction was applied to this edge. 


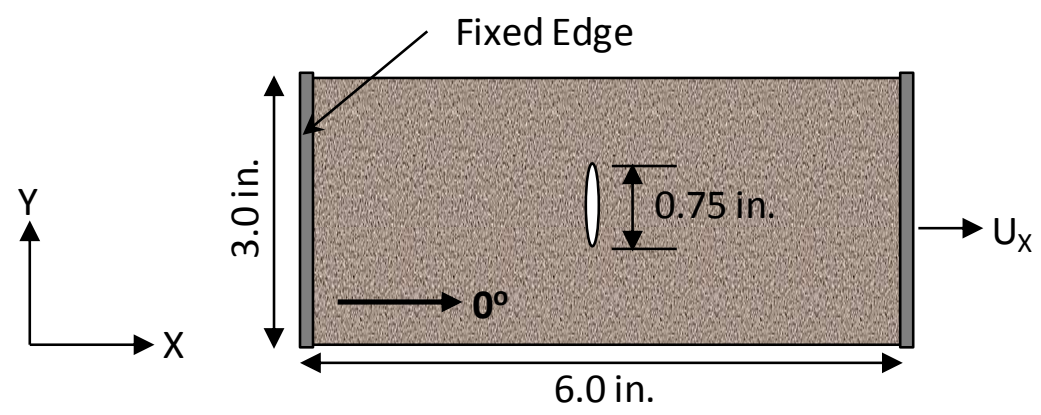

Figure 2. Schematic diagram of a center-notched coupon.

Three laminate configurations were considered in the study. The laminate thicknesses, stacking sequence and corresponding material system for each configuration are listed in Table 1. Mechanical properties and strengths of the material systems are provided in Table 2 .

Table 1. Laminate configurations.

\begin{tabular}{cccc}
\hline \hline ID & Laminate-A & Laminate-B & Laminate-C \\
\hline \hline Number of Plies & 10 & 16 & 34 \\
\hline Stacking Sequence & {$[45 / 0 /-45 / 0 / 90]_{\mathrm{s}}$} & $\begin{array}{c}{\left[45 / 90 /-45 / 0_{2} /-\right.} \\
45 / 90 / 45]_{\mathrm{s}}\end{array}$ & {$\left[45 / 0_{3} /-45 / 90 / 45 / 0_{3} /-45 / 90 / 45 / 0 /\right.$} \\
& 0.06 & 0.12 & 0.187 \\
\hline Thickness, inches & T800/3900-2 & IM7/8552 & IM7/PETI5 \\
\hline Material & &
\end{tabular}

Table 2. Material properties.

\begin{tabular}{cccccccccc}
\hline \hline $\begin{array}{c}\text { Material } \\
\text { System }\end{array}$ & $\begin{array}{c}\mathrm{E}_{11} \\
\times 10^{6} \mathrm{psi}\end{array}$ & $\begin{array}{c}\mathrm{E}_{22} \\
\times 10^{6} \mathrm{psi}\end{array}$ & $\begin{array}{c}\mathrm{G}_{12} \\
\times 10^{6} \mathrm{psi}\end{array}$ & $\begin{array}{c}\mathrm{X}_{\mathrm{t}} \\
\times 10^{6} \mathrm{psi}\end{array}$ & $\begin{array}{c}\mathrm{X}_{\mathrm{c}} \\
\times 10^{6} \mathrm{psi}\end{array}$ & $\begin{array}{c}\mathrm{Y}_{\mathrm{t}} \\
\times 10^{6} \mathrm{psi}\end{array}$ & $\begin{array}{c}\mathrm{Y}_{\mathrm{c}} \\
\times 10^{6} \mathrm{psi}\end{array}$ & $\begin{array}{c}\mathrm{S}_{12} \\
\times 10^{6} \mathrm{psi}\end{array}$ & $v_{12}$ \\
\hline IM7/8552 & 24.85 & 1.32 & 0.765 & 0.337 & 0.1740 & 0.0189 & 0.0289 & 0.0167 & 0.32 \\
\hline T800/3900-2 & 23.20 & 1.30 & 0.900 & 0.412 & 0.225 & 0.0138 & 0.0240 & 0.0168 & 0.28 \\
\hline IM7/PETI5 & 21.89 & 1.40 & 0.634 & 0.307 & 0.225 & 0.0090 & 0.0369 & 0.0145 & 0.34 \\
\hline \hline
\end{tabular}

Several finite element models of the coupon, shown in Fig. 2, were developed for simulating in-plane damage progression only. Delaminations between the plies were not included in the models. The finite element models of the coupon were uniformly discretized using the 2D quadrilateral S4R shell elements available in the ABAQUS ${ }^{9}$ element library. The S4R element is a reduced integration four noded shell element and has three translational and three rotational degrees of freedom at each node. The entire thickness of each laminate was represented by one shell element with one integration point at the element center for each layer. The element sizes used in the finite element models are 0.01 in. X 0.01 in., 0.02 in. X 0.02 in., 0.04 in. X 0.04 in., 0.06 in. X 0.06 in., 0.08 in. X 0.08 in., 0.1 in. X 0.1 in., 0.12 in. X 0.12 in., 0.14 in. X 0.14 in., 0.16 in. X 0.16 in. and 0.2 in. X 0.2 in. The notch in the coupon was modeled using coincident nodes at the notch location in the FEMs.

Progressive failure analyses (PFA) of these models were performed using the COSTR damage model in ABAQUS $^{\mathrm{TM}} /$ Explicit solver version 6.7-4. The failure load predictions obtained from the PFA are plotted in Fig. 3 as a function of element area for the three laminate configurations.. The actual analysis results are represented by the symbols and a $6^{\text {th }}$ order polynomial curve is fitted through these points. It can be noticed in Fig. 3, that these three curves follow a very similar trend where the failure load increases very sharply as the element area increases and reaches a constant value for elements with area greater than $0.026 \mathrm{in}^{2}$ (not shown). The constant failure load predicted when large elements are used could be attributed to the inability of the larger elements to capture the stress concentration at the notch tips. 


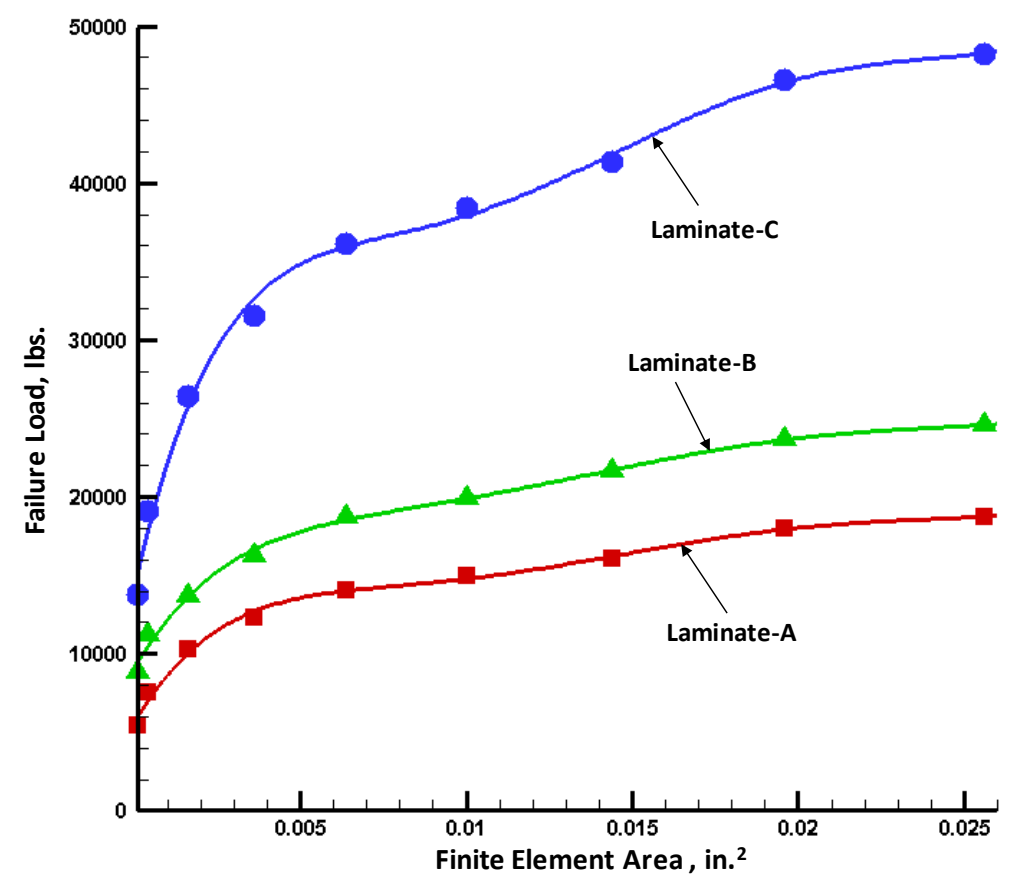

Figure 3. Failure load vs. element area for center-notched coupons.

The $6^{\text {th }}$ order polynomial equations of the curves representing the failure load as a function of finite element area in Fig. 3 are used to generate the curves in Fig. 4, after normalizing them with respect to their maximum load. The derivative curves represented by dot-dash lines in Fig. 4, provide insight into the sensitivity of failure load with respect to the change in element area and are obtained by differentiating the $6^{\text {th }}$ order polynomial equations of the curves with respect to the finite element area.

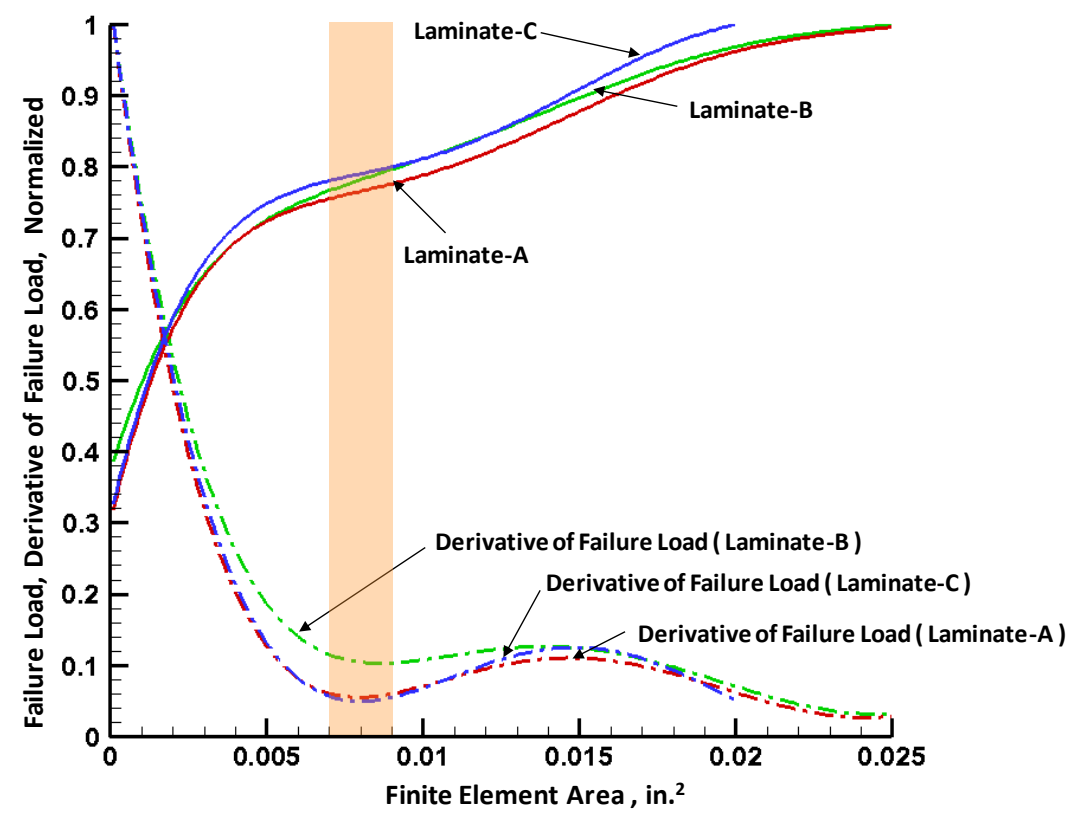

Figure 4. Normailzed failure load and its derivatives with respect to element area.

From these derivative curves, which have qualitatively similar shapes for all three laminates, it can be observed that for a small range of element area, around $0.007 \mathrm{in}^{2}$ to $0.009 \mathrm{in}^{2}$ shown as the vertical shaded bar, the change in failure load with change in element size is small. Additionally, for elements with area greater than 0.026 in. $^{2}$, the 
magnitude of the derivative curve reaches zero indicating a constant failure load. Based on the results shown in Figs. 3-4, elements with edge lengths equal to $0.09 \mathrm{in}$. were used in discretizing a finite element model of a quasiisotropic laminate [45/0/-45/90]s coupon shown in Fig. 2. The coupon is $0.048 \mathrm{in}$. thick and made from the T800/3900-2 material system. Boundary conditions, identical to those applied to the other coupons in this study, were applied to this coupon also. Progressive failure analysis of this coupon, performed using the COSTR damage model, predicted a failure load of $10395.6 \mathrm{lbs}$. verses an average experimental failure load of $10613.6 \mathrm{lbs}$. It can be observed here that the failure load prediction matches closely with the experimental data with a percent error of 2.1\%. Self-similar cracks were predicted for this coupon and the same were observed in the test. Hence a square element of size 0.09 in. X 0.09 in. will be used in discretizing the finite element models of the large notched laminated panels which are presented in the next section, and a PFA will be performed for residual strength predictions.

\section{Residual Strength of Large Notched Panels}

The element size identified in the previous section was used to develop finite element models for predicting damage progression and residual strength of large center-notched sandwich and laminated composite panels, loaded in compression and tension respectively. All of the large notched panels were fabricated and tested by The Boeing Company. The physical dimensions of the panels are related to the notch lengths and are listed in Table 3.

Table 3. Physical dimensions of large notch panels.

\begin{tabular}{c|c|c|c}
\hline $\begin{array}{c}\text { Panel } \\
\text { ID }\end{array}$ & $\begin{array}{c}\text { Width, } \\
\text { in. }\end{array}$ & $\begin{array}{c}\text { Length, } \\
\text { in. }\end{array}$ & $\begin{array}{c}\text { Notch Length, } \\
\text { in. }\end{array}$ \\
\hline SP1 & 10.0 & 30.0 & 2.0 \\
\hline SP2 & 20.0 & 40.0 & 4.0 \\
\hline SP3 & 50.0 & 110.0 & 10.0 \\
\hline LP1 & 50.0 & 110.0 & 10.0 \\
\hline \hline
\end{tabular}

Sandwich panels SP1, SP2 and SP3, each configured with two different face-sheet thicknesses, were considered. The face-sheets consisted of 10 plies and 32 plies respectively. The thinner face-sheet laminates were quasiisotropic. The thick face-sheet configuration with 32 plies forms a harder laminate where the percentage of $0^{\circ}$ plies was increased relative to the $45^{\circ}$ and $90^{\circ}$ plies. The core is $2.0 \mathrm{in}$. thick in all of the sandwich panels. In the case of the large notch laminated panels (LP1a and LP1b), one of the panels was made with 18 plies and the other panel was made with 22 plies. The face-sheets of the sandwich panels and the laminated panels are made of typical graphite epoxy material.

Finite element (FE) models of the large notch sandwich and laminated panels are shown in Fig. 5. The FE models are discretized with S4R shell elements and the intra-laminar progressive failure analyses were performed using the COSTR damage model. The entire thickness of the sandwich and laminated panels were represented by one shell element. In the sandwich panel models, the core was modeled as a ply in the laminate. The typical finite element models for a mid size and a large size panel are shown in Figs. 5a and 5b, respectively. The finite element shape in the vicinity of the notch tips is shown in Fig. 5c. In the case of panels with 10.0 in. notches, where the panel dimensions are large, discretizing the entire length of the panel with $0.09 \mathrm{in}$. X 0.09 in. size elements was not computationally viable. Hence the center region of the panel, where the damage is expected to initiate and propagate, was modeled with 0.09 in. X 0.09 in. elements and the remainder of the panel was modeled using larger size elements as shown in Fig. 5b. However for the 2.0 in. and 4.0 in. notch cases the entire model area was discretized using uniform size elements of size 0.09 in. X 0.09 in. 


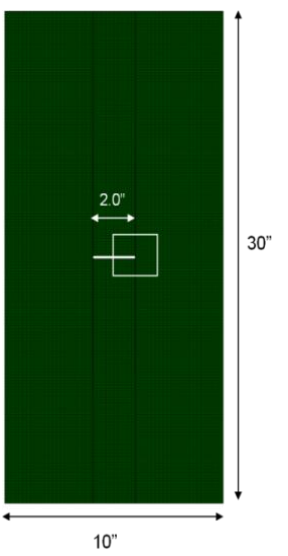

a) 2.0 in. Notched panel.

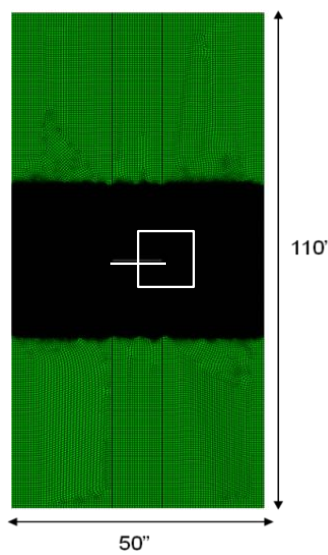

b) 10.0 in. Notched panel.

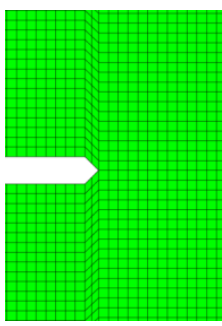

c) Notch tip region.

Figure 5. Finite element model, notched.

Progressive failure analyses (PFA) of the large notched panel models were performed using the COSTR damage model in ABAQUS ${ }^{\mathrm{TM}} /$ Explicit solver version 6.7-4. Since the explicit solver (which is a transient analysis solver) is employed to perform the quasi-static nonlinear analysis of the large notched panels, a mass density of 0.000154 lbs$\mathrm{sec}^{2} / \mathrm{in}^{4}$ for the composite material was used in the analysis. During the entire simulation, the kinetic energy of the model was monitored to ensure that it did not exceed 10 percent of the total strain energy of the model in accordance with the ABAQUS ${ }^{\mathrm{TM}^{9}}$ User's Manual guidelines for simulating quasi-static loading in the explicit finite element solver'. This guideline (maintaining the kinetic energy to a minimum while simulating a pseudo-static analysis) ensures that significant dynamic effects were not introduced while using a small total loading time required for computational efficiency in an explicit analysis.

\section{A. Large Notched Sandwich Panels Subjected to Compression Loading}

The failure loads obtained from the intra-laminar progressive failure analyses are presented in Table 4, along with the test data. Comparison of the predicted failure loads with the experimentally measured failure loads reveals a good agreement with a maximum percentage difference of $6.5 \%$.

Table 4. Physical dimensions of notched sandwich panels and the accuracy of predicted failure loads.

\begin{tabular}{c|c|c|c|c|c}
\hline \hline $\begin{array}{c}\text { Panel } \\
\text { ID }\end{array}$ & $\begin{array}{c}\text { Facesheet } \\
\text { Thickness } \\
\text { in number } \\
\text { of plies. }\end{array}$ & $\begin{array}{c}\text { Width } \\
\text { in. }\end{array}$ & $\begin{array}{c}\text { Length } \\
\text { in. }\end{array}$ & $\begin{array}{c}\text { Notch Length } \\
\text { in. }\end{array}$ & $\begin{array}{c}\text { Predicted } \\
\text { Failure Load } \\
\text { Error, \% }\end{array}$ \\
\hline SP1a & 10 & 10.0 & 30.0 & 2.0 & 6.0 \\
\hline SP1b & 32 & 10.0 & 30.0 & 2.0 & 6.3 \\
\hline SP2a & 10 & 20.0 & 40.0 & 4.0 & -4.7 \\
\hline SP2b & 32 & 20.0 & 40.0 & 4.0 & -2.2 \\
\hline SP3a & 10 & 50.0 & 110.0 & 10.0 & -4.9 \\
\hline SP3b & 32 & 50.0 & 110.0 & 10.0 & -5.3 \\
\hline \hline
\end{tabular}

A positive error sign means that the failure load was overpredicted, while a negative sign means that the predicted load was a little bit lower than the failure load demonstrated in the test. A self-similar crack path (crack 
parallel to the notch) was predicted for all six panels and the same was observed in the test. The failed test panel and the simulated crack path in the 10.0 in. notched sandwich panel SP3b are shown in Figs. 6a and 6b, respectively.

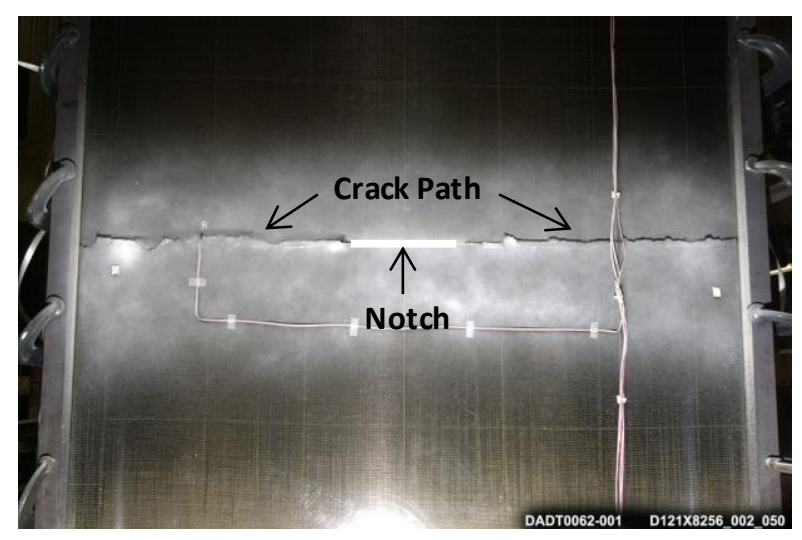

a) Test panel.

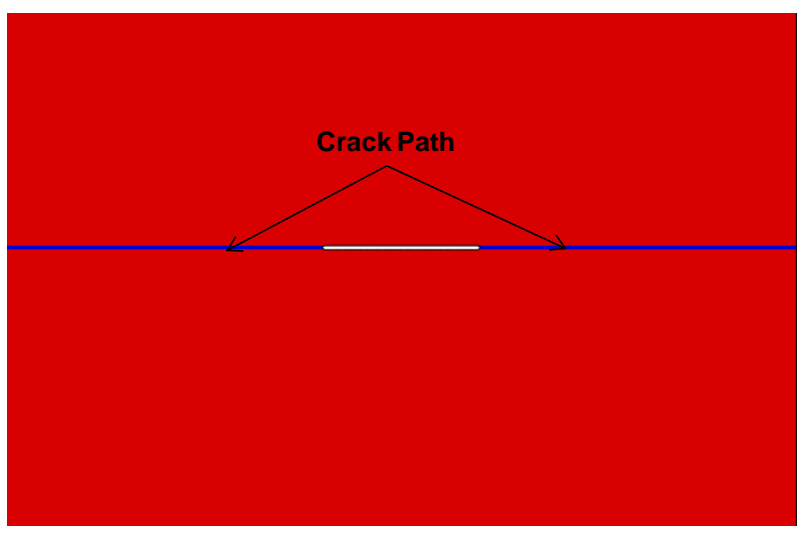

b) Finite element model.

Figure 6. Crack trajectories in a 10 in. notched sandwich panel.

\section{B. Large Notched Laminated Panels subjected to Tension Loading}

Predicted failure load, percent error and crack trajectories for large notched laminated panels subjected to tension loads are presented in Table. 5 and in Fig. 7, respectively. The predicted failure loads for these two panels is in good agreement with the test data and is generally, within $10 \%$ of the experimentally determined load. The suffix in the failure load for the second panel (LP1b) indicates that the loading during testing was stopped after the splitting cracks (cracks perpendicular to notch) developed and the panel was not loaded to complete failure. Similarly the PFA simulation was also stopped after the splitting cracks developed as it was sufficient to capture the splitting load and the crack trajectory for comparison with the test data.

Table 5. Physical dimensions of notched laminated panel and failure loads.

\begin{tabular}{c|c|c|c|c|c}
\hline \hline $\begin{array}{c}\text { Panel } \\
\text { ID }\end{array}$ & $\begin{array}{c}\text { Facesheet } \\
\text { Thickness } \\
\text { in number } \\
\text { of plies }\end{array}$ & $\begin{array}{c}\text { Width } \\
\text { in. }\end{array}$ & $\begin{array}{c}\text { Length } \\
\text { in. }\end{array}$ & $\begin{array}{c}\text { Notch Length } \\
\text { in. }\end{array}$ & $\begin{array}{c}\text { Predicted } \\
\text { Failure Load } \\
\text { Error, \% }\end{array}$ \\
\hline LP1a & 18 & 50.0 & 110.0 & 10.0 & -9.1 \\
\hline LP1b & 22 & 50.0 & 110.0 & 10.0 & +8.1 \\
\hline \hline
\end{tabular}

The crack paths that developed in the laminated panels during the test and the simulated crack paths are presented in Fig. 7. The crack paths simulated from the progressive failure analysis closely match the crack paths that were noticed in the test panels. The crack path of the failed test panel (LP1a) and the simulated crack path are shown in Figs. 7a and 7b, respectively. It can be noticed that self similar cracks (parallel to the notch direction) are simulated for LP1a and the same was observed in the test panel. However for LP1b, splitting cracks (perpendicular to the notch direction) were simulated by the PFA (see Fig. 7d) and similar crack paths were observed in the test panel (see Fig. 7c). In general, it has been observed that as the percentage of $0^{\circ}$ plies in a laminate becomes greater than $50 \%$, splitting cracks develop when the panel is subjected to tensile loading parallel to the $0^{\circ}$ direction. ${ }^{12}$ In the case of laminates where the percentage of $0^{\circ}$ plies is less than $50 \%$, self similar cracks are observed. 


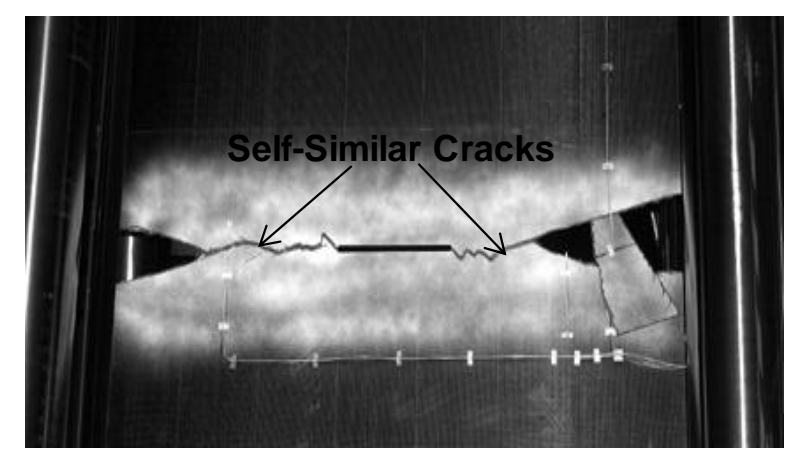

a) Experimentally observed crack path in panel LP1a.

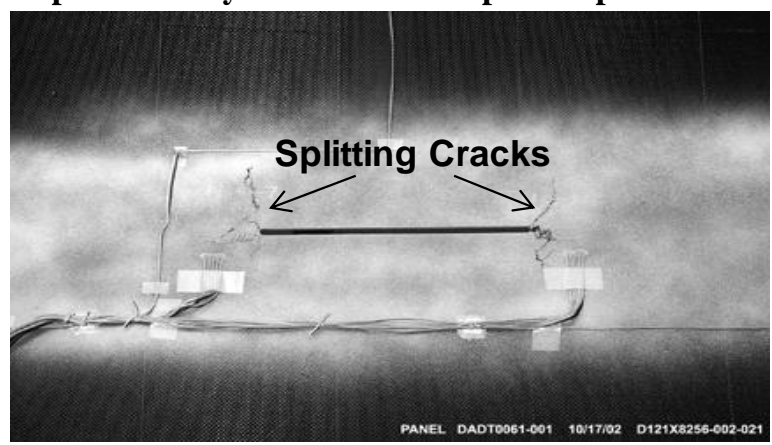

c) Experimentally observed crack path in panel LP1b.

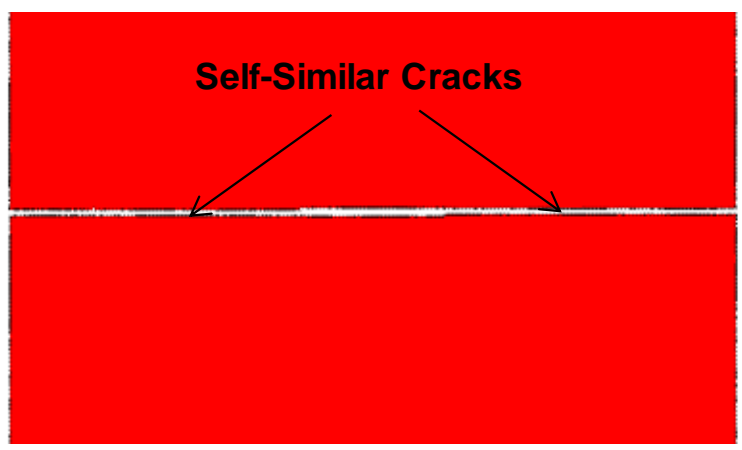

b) Predicted crack path in panel LP1a.

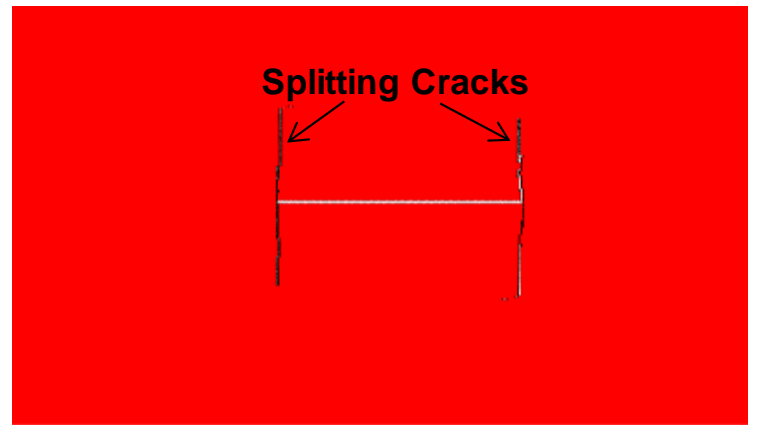

d) Predicted crack path in panel LP1b.

Figure 7. Experimentally observed and predicted crack trajectories in notched laminated panels, LP1a and LP1b.

\section{Development of Mesh Regularization Procedure Utilizing Characteristic Length Approach}

Most progressive failure analysis methodologies based on continuum damage mechanics exhibit a high degree of mesh dependency in predicting failure loads if mesh regularization procedures are not built in to the methodology. The sensitivity of predicted failure load to the spatial discretization was demonstrated in Section III for a center notched coupon loaded in tension. The reasons that the classical continuum damage mechanics models, implemented in the finite element analysis approach, are sensitive to the spatial discretization have been explained in detail in Ref. 2. Also a numerical procedure that leads to the conservation of finite element mesh objectivity, has been outlined in Ref. 2-4, 13. The numerical procedure involves the critical fracture energy of the material and a characteristic length, which in general is related to the width of the damage zone. Utilizing these key material parameters, the regularization process is accomplished by modifying the material strengths in an element based on its size, while preserving the critical fracture energy of the material as shown in the relationship below.

$$
X_{t}=\frac{2 G_{c}}{\varepsilon^{f} L_{E}}
$$

Where $X_{\mathrm{t}}$ is the failure stress, $L_{\mathrm{E}}$ is the characteristic length of the element, $\varepsilon^{f}$ is the failure strain and $G_{\mathrm{c}}$ is the critical fracture energy of the material ${ }^{1,4}$ for the fracture mode of interest. The utility of this approach is in the potential to increase element size by adjusting material strengths which potentially leads to computationally efficient models.

In our study, a method is proposed for mesh regularization based on a strength scaling factor for notched coupons where failure is dominated by brittle failure mode. In this approach, the solid red curve in Fig. 4, which represents the relationship between the normalized failure load and the element area for Laminate-A, is considered and a $6^{\text {th }}$ order polynomial equation of that curve is obtained as shown below. 


$$
F L(x)=A+B x+C x^{2}+D x^{3}+E x^{4}+F x^{5}+G x^{6}
$$

Where $x$ is the element area and the constants of the above equation are provided in Table 6 below.

Table 6. Coefficients in Eq. (9).

\begin{tabular}{c|c}
\hline \hline Constants & Values \\
\hline$A$ & 0.3007964 \\
\hline$B$ & $1.9152725 \mathrm{E}+2$ \\
\hline$C$ & $-3.3148561 \mathrm{E}+4$ \\
\hline$D$ & $2.941072 \mathrm{E}+6$ \\
\hline$E$ & $-1.3157617 \mathrm{E}+8$ \\
\hline$F$ & $2.85746 \mathrm{E}+9$ \\
\hline$G$ & $-2.390701 \mathrm{E}+10$ \\
\hline \hline
\end{tabular}

The strength scaling factor (SSF) is obtained as:

$$
S S F=\frac{F L\left(x^{*}\right)}{F L(x)}
$$

where $x^{*}$ is the reference characteristic area and $x$ is the actual element area in the finite element model. In our study, $0.0081 \mathrm{in}^{2}$ is considered as the reference characteristic area which corresponds to an element size of $0.09 \mathrm{in}$. $\mathrm{X} 0.09$ in. This $S S F$ is then utilized as a multiplier to all of the strength parameters to obtain the modified material strengths corresponding to the actual element area in the finite element model. A small variation in the aspect ratio of an element (within 5\%) should still yield an acceptable solution as long as the area is close to the reference characteristic area. At this point, the mesh regularization procedure is not performed on non square elements and the authors intend to do so in the near future. The above procedure was implemented in the user-subroutine VUMAT along with the COSTR damage model and its capabilities are demonstrated in the next section.

\section{Mesh Regularization of a Notched Coupon}

A preliminary verification of the mesh regularization procedure presented in the previous section is performed by applying it to the center notched coupon shown in Fig. 2. Laminate-A in Table. 1 is chosen as an example for the demonstration of the regularization procedure. The finite element models of the coupon, shown below, are developed and analyzed with the intention of showing the convergence of failure load for varying element sizes for coupons which fail in a self-similar fashion. The interaction of other damage modes, such as delamination, on the strength scaling procedure will be investigated in future work.

Three FE models of the coupon were developed using S4R shell elements. The element sizes for the three models were approximately 0.04 in. X 0.04 in. (Model A), 0.1 in. X 0.1 in. (Model B), and 0.2 in. X 0.2 in. (Model C). A quarter section of each model is shown in Fig. 8 even though the full model of the coupon was considered in the PFA. One edge of the coupon is fixed in all degrees of freedom (dof) and a uniform edge displacement is applied to the other edge of coupon which is free to move in the loading direction only. A detailed explanation of the model and the boundary conditions is explained in Section. III. 


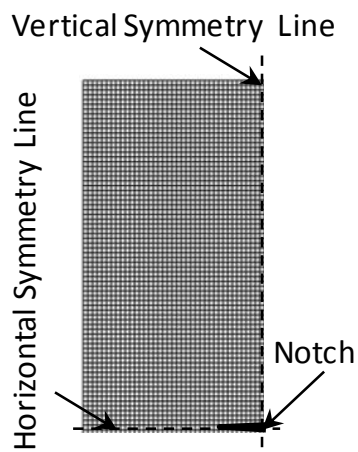

a) Finite Element

Model A.

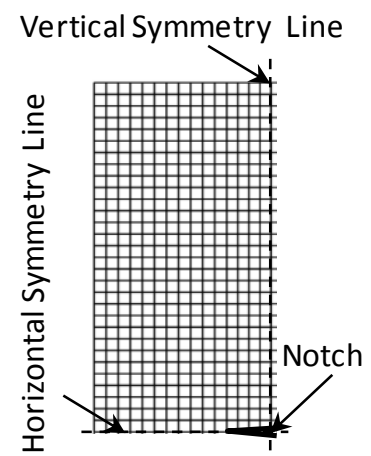

b) Finite Element

Model B.

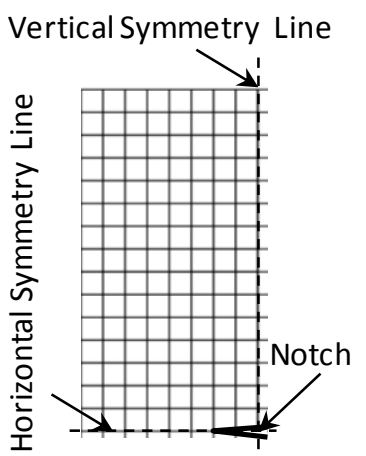

c) Finite Element Model C.

Figure 8. Quarter section of finite element models of varying element sizes.

The progressive failure analyses of the above models were performed with the COSTR damage model. In Fig. 9a the failure load vs. edge displacement curves for the three models A-C are presented. These results were obtained from the PFA analyses without the mesh regularization procedure and it is readily seen in the figure that the predicted failure load increases with increase in element size. In Fig. 9b, failure load vs. edge displacement curves of the same three models A-C with the mesh regularization procedure implemented in the PFA are presented. It can be noticed that all the three models with different size elements provide nearly the same failure load and the axial edge displacement.

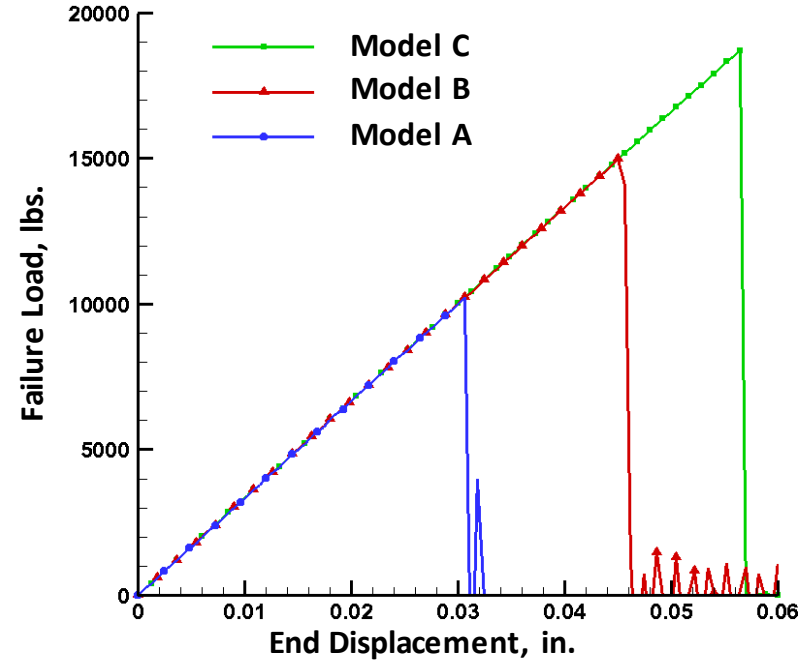

a) Without mesh regularization procedure.

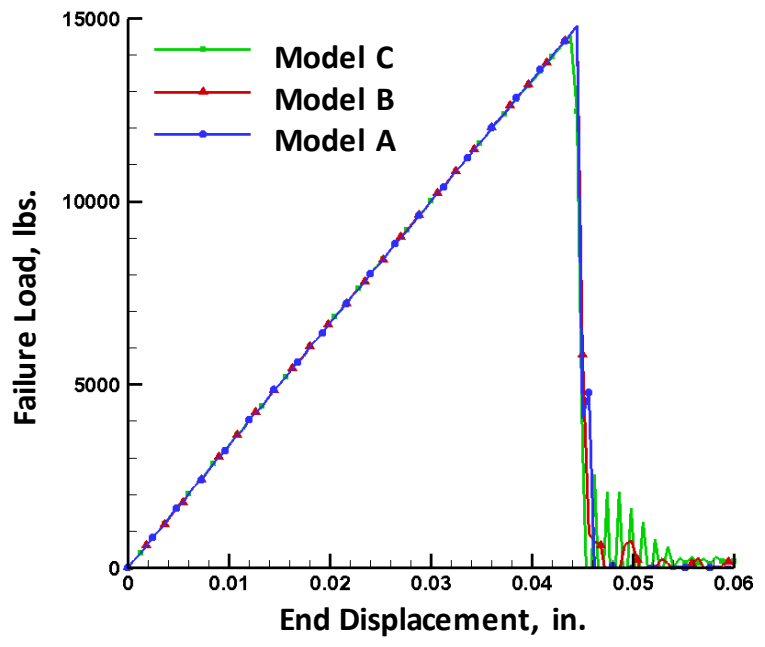

b) With mesh regularization procedure.

Figure 9. Effect of element size and mesh regularization on failure load predictions, Laminate A.

\section{Conclusion}

A complete stress reduction (COSTR) damage model has been outlined along with a finite element modeling procedure to perform progressive failure analysis of large notched laminated and sandwich panels. The numerical approach employed in determining an element size for performing PFA of notched coupons, which fail in a brittle fashion has been discussed. The element size determined from the study, $0.09 \mathrm{in}$. X $0.09 \mathrm{in}$., was independent of the material system and layup for the panels considered. This element size, was close to but slightly larger than the size used previously by the authors in simulating experimental results with their COSTR PFA methodology. ${ }^{6}$ The numerically determined element size has been used in performing PFA of a number of notched laminated and 
sandwich panels tested by the Boeing Company. A good agreement between predicted and experimentally determined failure loads and the crack paths was obtained. The percentage error between the predicted and experimentally determined failure load is within $+/-6.5 \%$ for the notched sandwich panels and around $9.0 \%$ for the notched laminated panels. Even though some of the Boeing notched laminated coupons (not presented here) were analyzed previously with the old element size with good correlation with the experiments in most cases, in a small percent of cases the percent error was larger than observed currently. When these specimens were reanalyzed using the newly determined element size the results improved to within an acceptable range and correct damage paths were predicted, in some cases self-similar notch extension and in other cases splitting perpendicular to the notch tips.

Finally, an alternative approach for mesh regularization, using the element size determined in Section III as a "characteristic reference area" was developed for coupons that fail in a brittle failure mode. This approach scaled the failure strength, based on the ratio of the reference normalized load corresponding to the reference area, determined from the element size vs. failure load relationship, with the normalized load determined for the desired element area. Preliminary results indicate a good convergence of failure loads with respect to that corresponding to the reference element size. These results demonstrate a potential for using larger elements in performing PFA which will lead to enhanced computational efficiency.

\section{References}

${ }^{1}$ Bazant, Z. P., Oh, B. H., “CrackBand Theory for Fracture of Concrete," Materials and Structures, Vol. 16, No. 3, 1983, pp 155-177.

${ }^{2}$ Brekelmans, W.A.M., Vree, J.H.P., "Reduction of mesh sensitivity in continuum damage mechanics," Acta Mechanica, No. 110, pp 49-56, 1995.

${ }^{3}$ Camanho, P.P., Maimi, P., Davila, C.G., "Prediction of size effects in notched laminates using continuum damage mechanics," Composites Science and Technology, No. 67, pp 2715-2727, 2007.

${ }^{4}$ Lapczyk, I., Hurtado, J. A., "Progressive damage modeling in fiber-reinforced materials," Composites: Part A, No. 38, pp 2333-2341, 2007.

${ }^{5}$ Coats, T.W., and Harris, C.E., "A Progressive Damage Methodology for Residual Strength Predictions of Notched Composite Panels," NASA/ TM-1988-207646.

${ }^{6}$ Bogert, B. P., Satyanarayana, A., and Chunchu, B. P.: "Comparison of Damage Path Predictions for Composite Laminates by Explicit and Standard Finite Element Analysis Tools," Proceedings of the $47^{\text {th }}$ AIAA/ASME/ASCE/AHS/ASC Structures, Structural Dynamics and Materials Conference, Newport, Rhode Island, May 2006, AIAA 2006-1750.

${ }^{7}$ Satyanarayana, A., Przekop, A., "Predicting Failure Progression and Failure Loads in Composite Open-hole Tension Coupons," NASA / CR-2010-216700.

${ }^{8}$ Song, K.; Li, Y.; and Rose, C. A., "Continuum Damage Mechanics Models for the Analysis of Progressive Failure in Open-Hole Tension Laminates," Proceedings of the $52^{\text {nd }}$ AIAA/ASME/ASCE/AHS/ASC Structures, Structural Dynamics and Materials Conference, April 2011, Denver, Colorado, AIAA 2011-1861.

${ }^{9}$ Anon., ABAQUS ${ }^{\text {TM }}$ User's Manual, Vol. 1-3, Version 6.5. Hibbitt, Karlsson, and Sorensen, Pawtucket, Rhode Island, 2003.

${ }^{10}$ Knight, N. F., Jr., "Factors Influencing Progressive Failure Analysis Predictions for Laminated Composite Structure," Proceedings of the $49^{\text {th }}$ AIAA/ASME/ASCE/AHS/ASC Structures, Structural Dynamics, and Materials Conference, Schaumburg, Illinois, April 2008, AIAA-2008-2108.

${ }^{11}$ Hashin, Z., and Rotem, A., "A Fatigue Failure Criterion for Fiber-Reinforced Composite Materials," Journal of Composite Materials, Vol. 7, October 1973, pp. 448-464.

${ }^{12}$ Askari, E., Xu, J., \& Silling S., "Peridynamic Analysis of Damage and Failure in Composites," Proceedings of the $44^{\text {th }}$ AIAA Aerospace Science Meeting and Exhibit, Reno, Nevada, January 2006, AIAA-2006-88.

${ }^{13}$ Bazant, Z. P., Cabot, P. G., "Measurement Of Characteristic Length of Nonlocal Continuum," Journal of Engineering Mechanics, Vol. 115, No. 4, April, 1989, pp. 755-767. 